\title{
An extinction trial as a reminder treatment following electroconvulsive shock
}

\author{
WILLIAM C. GORDON and ROBERT R. MOWRER \\ University of New Mexico, Albuquerque, New Mexico 87131
}

\begin{abstract}
Animals given electroconvulsive shock (ECS) following active avoidance training were found to exhibit poor retention of the active avoidance response. However, this deficit was alleviated if the animals received an extinction trial for active avoidance prior to retention testing. That the trial was an extinction trial was demonstrated by the fact that exposure to the trial decremented the performance of animals not given ECS after learning. The implications of these findings for explanations of retrograde amnesia are discussed.
\end{abstract}

Retrograde amnesia produced by electroconvulsive shock (ECS) usually has been attributed to a failure in memory storage or consolidation processes (cf. McGaugh, 1966; McGaugh \& Dawson, 1971). According to this view, the information from a learning trial undergoes gradual storage processing following that trial, and this processing is necessary in order for the information to be registered in long-term or permanent memory. The postlearning administration of ECS is presumed to disrupt these processes, resulting in a permanent loss of the information from storage. It is hypothesized, then, that animals perform poorly on post-ECS retention tests because of the absence of training information.

Recently, a number of experiments employing "reminder paradigms" have called this explanation of retrograde amnesia into question. In these experiments, animals learned to perform some response and then received ECS, as in a normal retrograde amnesia experiment. Prior to retention testing, however, the animals were confronted with some portion of the stimulus complex that was present during learning (the reminder treatment). Since many of these experiments involved training with aversive stimuli, the reminder treatments often consisted of a noncontingent reexposure to the aversive stimulus used in training (e.g., Miller \& Springer, 1972). However, reexposure to the training context has also been used successfully as a reminder treatment (e.g., Miller, Ott, Berk, \& Springer, 1974). It is clear from these experiments that reexposure to training stimuli prior to testing can alleviate the amnesia normally associated with ECS administration (Lewis, 1976; Miller \& Springer, 1973; Spear, 1978).

These reminder studies have been interpreted as posing serious problems for a storage failure explanation of ECS-induced amnesia. Since the storage failure position maintains that ECS causes a loss of information

This research was supported by a grant from the National Science Foundation to the first author. Reprint requests should be sent to William C. Gordon, Department of Psychology, University of New Mexico, Albuquerque, New Mexico 87131. from storage, this model must predict that the resulting amnesia should be permanent. According to this view, retention following ECS should be restored only if additional learning occurs to take the place of the lost information. Yet, in the reminder studies, forgetting is reduced by treatments that cannot readily be labeled reacquisition trials and that often take place outside the training apparatus. This has led some researchers to suggest that ECS causes amnesia by interfering with processes important for information retrieval, not by eliminating information from storage (e.g., Lewis, 1976; Miller \& Springer, 1973). These researchers suggest that if forgetting can be reduced by reminder cues, then the information necessary for good retention must still be present in storage at the time of cuing. Thus, the amnesia normally produced by ECS must result from an animal's inability to retrieve or use the information that is available in storage.

Although these reminder studies seem to represent strong evidence that ECS does not interrupt information storage, there have been efforts to reconcile such findings with the storage failure hypothesis. Gold and King (1974) have argued, for example, that the improved retention induced by reminder treatments can be traced to a combination of two factors. First, in many reminder experiments, the ECS intensities used have been relatively low, resulting in incomplete amnesias. Thus, it is reasonable to assume that in many reminder experiments, animals have retained at least some residual information about training after administration of the ECS. Second, although most of the reminder treatments have not consisted of actual retraining trials, the treatments do constitute opportunities to acquire new information. Furthermore, because of the similarity of training and reminder stimuli, the new learning that occurs is often compatible with the information acquired during original training. By being compatible, it is meant that the new information acquired during the reminder treatment would transfer positively to any retention test for the originally learned response.

It is clear from the control conditions used in the 
reminder studies that neither of these factors aloneresidual information after ECS or new information from reminder treatments-produce the good retention found in the reminder studies (e.g., Miller \& Springer, 1972). However, Gold and King (1974) suggest that it is the combination of these factors that produces improved retention in ECS-treated animals. Thus, according to this interpretation, ECS produces a partial loss of information from storage, but the lost information is replaced by compatible information acquired during the reminder treatment.

Although there has been evidence supporting the plausibility of this interpretation (e.g., Gold, Haycock, Macri, \& McGaugh, 1973), there have been few attempts to test directly this explanation of reminder effects. Any such test would require determining whether or not reminder effects occur in the absence of either residual information or new compatible learning. The present experiment represents an attempt to make such a determination by using a reminder treatment that should contain incompatible rather than compatible information. In essence, animals are administered ECS following learning and are then reminded with an extinction trial for that prior learning. Previous experiments in our laboratory have shown that under some conditions, an extinction trial can improve the retention performance of animals after long retention intervals (Gordon, Smith, \& Katz, 1979). If it can be demonstrated that an extinction trial functions to improve the performance of an amnesic animal, this would indicate that a reminder treatment need not replace lost information with new compatible information in order to improve retention. Such a finding would represent strong evidence against a storage failure interpretation of ECSinduced amnesia.

\section{METHOD}

\section{Subjects and Apparatus}

The subjects were 90 male albino rats of the Sprague-Dawley strain, 50-75 days old at the time of original training. The primary apparatus was a one-way avoidance box consisting of one white translucent chamber and one black chamber. The chambers, each $27 \times 13 \times 13 \mathrm{~cm}$, were separated by a door that could be lowered (opened), leaving a $3-\mathrm{cm}$ hurdle between the chambers and activating a $2-\mathrm{Hz}$ flashing light $(7.5 \mathrm{~W})$ located behind the white chamber. The flashing light could be terminated by breaking a photobeam located $13 \mathrm{~cm}$ into the black chamber. The entire avoidance apparatus contained a grid floor made of .6 -cm-diam steel rods set $1.7 \mathrm{~cm}$ apart. A scrambled footshock provided by a Grason-Stadler shock generator (Model E1064GS) could be delivered through the grid floor underlying the white chamber. Located adjacent to the avoidance apparatus was an ECS generator (Lafayette Instruments, A615B) with leads that terminated in flattened alligator clips that could be attached to a rat's pinnae. The generator was set to deliver a $50-\mathrm{mA}$ shock for $500 \mathrm{msec}$.

In addition to the avoidance apparatus, a clear Plexiglas footshock chamber $(36 \times 36 \times 69 \mathrm{~cm})$ and a white footshock chamber, identical to the white chamber in the avoidance apparatus, were used. The floors in these chambers were identical to those in the avoidance apparatus, and footshocks could be delivered through these floors by the same shock source used for the avoidance apparatus. Wire-mesh holding cages were used to house animals during all intertrial intervals, all training-ECS intervals, and all reminder-test intervals.

\section{Design and Procedure}

Following $24 \mathrm{~h}$ of food deprivation and $5 \mathrm{~min}$ of handling, each animal received original training followed $72 \mathrm{~h}$ later by a retention test. There were eight primary treatment groups $(\mathrm{N}=10)$ derived from the factorial combination of two types of original training [active avoidance (AA) or noncontingent footshocks (FS)], the presence (ECS) or absence (sham) of ECS following training, and the presence (R) or absence (NR) of a reminder treatment prior to retention testing.

Active avoidance training (AA) consisted of placing an animal in the white chamber of the avoidance apparatus and, after $3 \mathrm{sec}$, lowering the chamber door. If an animal failed to cross over into the black chamber and break the photobeam within $5 \mathrm{sec}$ after the door was lowered, a .5-mA footshock was delivered and remained on until an escape response occurred. Following an escape response, the chamber door was closed and $3 \mathrm{sec}$ later the animal was removed to a holding cage for a $30-\mathrm{sec}$ intertrial interval (ITI). If an animal did cross into the black compartment within $5 \mathrm{sec}$ after the chamber door opened (an avoidance response), the door was closed and after $3 \mathrm{sec}$ the animal was removed for the ITI. The criterion for AA acquisition was three consecutive shock avoidances, which had to be achieved within 30 trials.

Each animal given noncontingent footshocks (FS) received the same number, sequence, and duration of footshocks given a randomly selected animal from the AA condition. However, these footshocks were inescapable and were given in the white footshock chamber outside the avoidance apparatus. Like the AA animals, each FS animal spent a $30-\mathrm{sec}$ ITI in a holding cage after each trial.

The AA or FS animals that received ECS were removed from either the avoidance apparatus or the white footshock chamber immediately after the last training trial. The flattened alligator clips were attached to the rats' pinnae, the animals were placed in a holding cage, and ECS was administered. In all cases; ECS administration occurred within $10 \mathrm{sec}$ of the end of the last training trial. Sham animals were treated identically to the ECS animals except that no current was delivered. Following either ECS or a sham treatment, each animal was returned to its home cage for the $72 \mathrm{~h}$ retention interval.

Animals that received the reminder treatment (R) were returned to the experimental room $16 \mathrm{~min}$ prior to retention testing and were placed in the white chamber of the avoidance apparatus for $60 \mathrm{sec}$ with the flashing light activated. The door separating the chambers was not opened and no shock was administered during the treatment. In essence, this treatment is the same as a response-blocking or response-prevention trial often used to extinguish active avoidance responding (e.g., Gordon, Smith, \& Katz, 1979). After the reminder treatment, each animal spent $15 \mathrm{~min}$ in a holding cage prior to retention testing. Animals in the NR condition received no reminder treatment and were brought to the experimental room at the time of retention testing.

The retention test consisted of five trials, identical to those given the AA animals during training except that no shocks were administered during testing. On each trial, the latency of an animal to cross into the black compartment was recorded. If an animal remained in the white compartment for $60 \mathrm{sec}$, the trial was terminated. Following a crossover or a $60-\mathrm{sec}$ latency, each animal spent an ITI of $30 \mathrm{sec}$ in a holding cage.

Aside from the eight primary treatment groups described, one additional group (baseline) was used. This group $(N=10)$ was treated identically to the FS-sham-NR group except that the noncontingent footshocks were administered in the large, clear 
Plexiglas chamber rather than in the white footshock chamber. This group was included to determine the crossover latencies of animals that had previously been shocked but had received no exposure to a white chamber and no contingency between shock and responding.

\section{RESULTS}

The mean number of trials necessary for the animals in the four $\mathrm{AA}$ groups to reach the active avoidance criterion was as follows: AA-ECS-R, 8.7; AA-ECS-NR, 11.2; AA-sham-R, 9.3; and AA-sham-NR, 8.9. An analysis of variance performed on these data revealed no significant differences $[F(3,36)<1]$, indicating that these groups did not differ in the degree of active avoidance acquisition prior to the postlearning treatments.

To assess retention test performance, two measures were analyzed. The first measure, latency to cross over into the black compartment on the first retention test trial, is represented in Figure 1 as a function of treatment condition. These latencies were converted to logs for purposes of statistical analysis. An analysis of variance was performed on the data from the eight main treatment groups, with the variables of interest being type of original training (training), presence or absence of ECS (ECS), and presence or absence of a reminder treatment (reminder). This analysis revealed a significant effect of the ECS variable $[F(1,72)=4.73, p<.05]$, as well as a significant ECS by Reminder Variable interaction $[F(1,72)=5.37, p<.05]$. The interaction of training, ECS, and reminder variables did not attain significance. Despite the failure to obtain a significant three-way interaction, individual analyses were performed on the AA and FS animals. The analysis of the AA condition data revealed a significant interaction between the ECS and reminder variables $[F(1,36)=6.42, p<.01]$. Critical-differences tests (Lindquist, 1956) were used to compare the individual treatment conditions. These tests showed that the ECS-NR animals had significantly

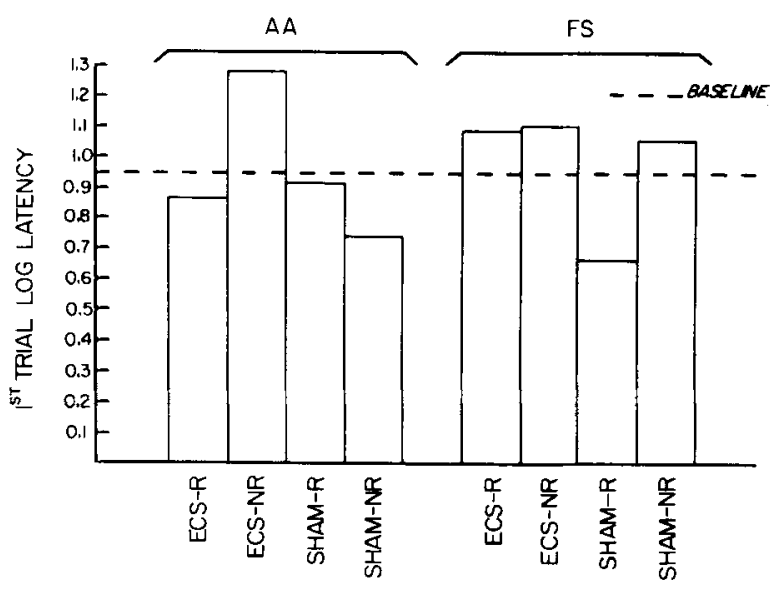

Figure 1. Mean log latencies on the first retention test trial for each of the treatment conditions. The dashed line represents the mean performance of the baseline condition animals. longer latencies than both the sham-NR and the ECS-R animals $(p<.05$, in both cases). These differences suggest that ECS produced a significant deficit in the retention of AA training and that the reminder treatment alleviated that deficit. However, this conclusion is tempered by the results of $t$ tests used to compare each of the AA groups with the baseline group. These comparisons revealed no significant differences between any AA treatment group and the baseline group on the first retention test trial.

The analysis of the FS condition data also revealed a significant ECS by Reminder interaction $[F(1,36)=$ 7.53, $\mathrm{p}<.05]$. However, critical difference tests showed that the interaction resulted from a pattern of effects different from that in the AA condition. Among the FS groups, the animals in the sham- $R$ group exhibited significantly shorter latencies than animals in any of the other three conditions ( $p<.05$, in all cases). As in the AA condition, however, $t$ tests revealed no significant differences between any FS group and baseline animals.

The second measure of retention used was the mean latency for the five retention test trials. The means of these average latency scores for each of the treatment conditions are represented in Figure 2. Again, these mean latency scores were converted to logs for purposes of analysis. An analysis of variance performed on the eight main treatment groups revealed a significant interaction of the reminder and the ECS variables $[F(1,72)=5.61$, $\mathrm{p}<.05]$. More importantly, the test revealed a significant three-way interaction of training, ECS, and reminder $[F(1,72)=6.02, p<.05]$. To assess the basis of this interaction, separate analyses were conducted on the $\mathrm{AA}$ and $\mathrm{FS}$ animals.

The analysis of the AA data revealed a significant ECS by Reminder interaction $[F(1,36)=15.97, p<.01]$. Critical-difference tests showed that the reminder treatment produced different patterns of performance in the ECS and sham conditions. The ECS-R animals exhibited significantly shorter latencies than either the ECS-NR or the sham-R animals ( $p<.05$, in both cases). However, both the sham-R and the ECS-NR animals showed significantly longer latencies than the sham-NR animals $(\mathrm{p}<.05$ and $\mathrm{p}<.01$, respectively).

These results clearly suggest that the ECS produced amnesia for active avoidance learning (cf. Groups shamNR and ECS-NR). Also, these results indicate that the reminder treatment reduced this amnesia (cf. Groups ECS$\mathrm{R}$ and ECS-NR). Importantly, these findings also show that the reminder treatment reduced the performance of animals not given ECS (cf. Groups sham-R and sham-NR). These conclusions are supported by $t$ tests comparing the AA treatment groups with the baseline group, which showed that the ECS-NR group had significantly longer latencies than the baseline group $(\mathrm{t}=2.63, \mathrm{p}<.05)$. However, both the ECS-R group and the sham-NR group exhibited significantly shorter latencies than did the baseline group $(\mathrm{t}=2.89, \mathrm{p}<.05$, and $\mathrm{t}=3.26, \mathrm{p}<.05$, respectively). 


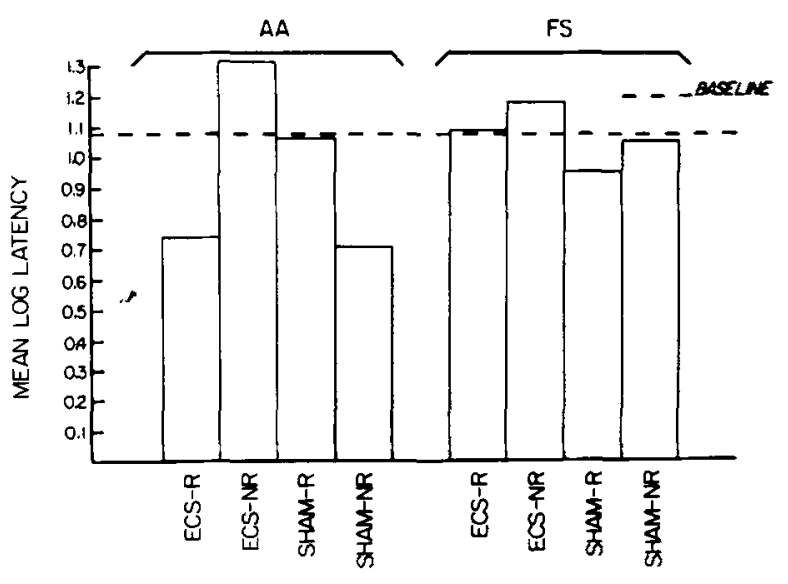

Figure 2. Mean log of the average latencies for all five retention test trials for each of the treatment conditions. The dashed line represents the mean performance of the baseline condition animals.

Finally, an analysis of variance performed on the mean latencies of animals in the FS condition revealed no significant differences among groups. Likewise, $t$ tests showed that none of the FS treatment groups differed significantly from the baseline group. These results suggest that the performance effects of ECS and reminder treatments found in the AA animals depended on prior avoidance learning and did not occur in animals previously exposed only to comparable shocks and apparatus cues.

\section{DISCUSSION}

Several conclusions can be drawn from these findings. First, it is clear that in the present study, ECS interfered with retention of the active avoidance training. Second, it is equally clear that the amnesia produced by ECS was alleviated by the reminder treatment used. Both of these conclusions receive some support from the first test trial data but are much more evident in the data from all five retention test trials. The third conclusion, which is of special significance in the present context, concerns the effects of the reminder treatment on animals not given ECS. It was demonstrated that although the reminder treatment improved the performance of ECS-treated animals, the treatment significantly reduced the performance of animals not exposed to ECS. This finding indicates that the information contained in the reminder treatment was not compatible with the information acquired during avoidance learning. In essence, the reminder treatment affected the performance of nonamnesic animals much as one would expect an extinction trial to do. Finally, these data show that the effects of ECS and the reminder treatment were not simply proactive effects on retention performance. These effects occurred only when animals had been exposed previously to active avoidance contingencies.

These findings represent strong evidence against a storage failure explanation of ECS-induced amnesia.
Contrary to the argument of Gold and King (1974), it is possible, apparently, to alleviate such amnesia without supplying an animal with additional information that is compatible with prior learning. In the absence of such additional compatible information, a storage failure hypothesis would have to predict that ECSinduced amnesia should be permanent. This does not appear to be the case.

Aside from representing evidence against the storage failure hypothesis, the present findings raise two questions that are related to some degree. The first concerns the mechanism by which reminder treatments alleviate amnesia, if, as the present findings suggest, they do not work simply by supplying new, compatible information. The second question concerns how one can explain the somewhat paradoxical finding that an extinction trial can enhance the performance of an amnesic animal while decreasing the performance of a nonamnesic animal.

The first of these questions is presently under investigation in our laboratory. We have suggested previously that poor retention may often result from the natural change that occurs in an organism's context or surroundings over time (cf. Feldman \& Gordon, 1979; Gordon \& Feldman, 1978). The consequences of this change would be that the stimuli, and thus the retrieval cues, that are available to an organism on a retention test bear little resemblance to those stimuli that had been present during learning and that had been stored as part of an organism's memory of training. This mismatch between retrieval cues and stored information would be expected to produce poor retention performance because of a retrieval failure (cf. Spear, 1973). Thus, it is possible that a reminder treatment functions to reduce this mismatch. In effect, we have suggested that a reminder treatment causes an organism to retrieve and reprocess previously stored information in a new, more contemporary context and that this new context becomes incorporated into the memory of prior training. Since the reminder treatment occurs after training, it is assumed that there will be greater similarity between the reminder and test contexts than between the learning and test contexts. Thus, the presence of the reminder contextual cues within a memory should facilitate subsequent retrieval.

As to the seemingly paradoxical effects of the extinction trial in the present study, we offer the following hypothesis. An extinction trial serves two functions. First, such trials contain cues from prior training and thus serve as reminders of prior learning. As such, extinction trials should serve to strengthen the retention performance for prior training. The second function of an extinction trial is to provide an organism with new information (a new contingency) that is incompatible with the information stored during learning. This incompatibility should result ultimately in a reduction in the performance of prior training.

We assume that these two functions of the extinction 
trial occur sequentially. In other words, we assume that it is necessary for an animal to retrieve a stored memory (the cuing function) before that memory can be modified (the performance reduction function). Following these assumptions, the degree to which an extinction trial of a given duration produces a performance increment or decrement should depend partially on how accessible a memory is at the time of cuing. If a memory is easily accessible (as in our sham animals), the extinction trial should result in fast retrieval and animals still should have additional exposure time to the trial in which to learn the incompatible contingency. The net result should be a decrement in retention performance. However, if a memory is difficult to retrieve (e.g., following ECS or a long retention interval), the cuing function should take longer, leaving an animal less exposure time in which to learn the incompatible contingency. The net result of this circumstance should be improved performance compared with that of a nonreminded animal. This hypothesis predicts the effects of the extinction trial in the present study as well as previous findings concerning exposure to extinction trials after short and long retention intervals (Gordon, Smith, \& Katz, 1979).

One additional question concerning the present data should be mentioned. It would seem reasonable to predict that the nonshock retention test trials themselves would serve as reminder treatments. If this were the case, one would predict that after a few such test trials, the performance of the NR animals should be similar to that of the $\mathrm{R}$ animals. However, the present data reveal no consistent changes in the performance of the NR animals over the five test trials used. One possible reason for this finding is that, in many cases, animals spent much less than $60 \mathrm{sec}$ in the white chamber of the apparatus on any given test trial. Thus the failure of the test trials to "remind" these animals may have been due to insufficient exposure to the apparatus cues during testing. However, it is also possible that, in order for cuing to be effective, an animal needs an uninterrupted period of time between a cue exposure and a subsequent test trial. In the present case, the $\mathrm{R}$ animals received $15 \mathrm{~min}$ in a holding cage between cuing and testing, while all animals received only $30 \mathrm{sec}$ between each retention test trial. The failure of retention test trials to remind animals is fairly common, and the basis of this failure deserves experimental attention.

Thus, the present findings provide new information concerning the possible effects of reminder treatments as well as the possible causes of ECS-induced amnesia. More importantly, however, these findings raise important questions concerning how information is processed by infrahumans. Further research into the mechanisms of reminder treatments should provide answers to some of these critical questions.

\section{REFERENCES}

Feldman, D. T., \& Gordon, W. C. The alleviation of short-term retention decrements with reactivation. Learning and Motivation, 1979, 10, 198-210.

Gold, P. E., Haycock, J. W., Macri, J., \& McGaugh, J. L. An explanation of the reminder effect: A case for retrograde amnesia. Science, 1973, 180, 1199-1201.

Gold, P. E., \& KING, R. D. Retrograde amnesia: Storage failure versus retrieval failure. Psychological Review, 1974, 81, 465-469.

Gordon, W. C., \& Feldman, D. T. Reactivation-induced interference in a short-term retention paradigm. Learning and Motivation, 1978, 9, 164-178.

Gordon, W. C., Smith, G. J., \& Katz, D. S. Dual effects of response blocking following avoidance learning. Behavior $R e$ search and Therapy, 1979, 17, 479-487.

LEwIS, D. J. A cognitive approach to experimental amnesia. American Journal of Psychology, 1976, 89, 51-80.

LINDQUIST, E. F. Design and analysis of experiments in psychology and education. Boston: Houghton Mifflin, 1956.

McGaugh, J. L. Time-dependent processes in memory storage. Science, 1966, 153, 1351-1358.

McGaugh, J. L., \& Dawson, R. G. Modification of memory storage processes. In W. K. Honig \& P. H. R. James (Eds.), Animal memory. New York: Academic Press, 1971.

Miller, R. R., Ott, C. A., Berk, A. M., \& Springer, A. D. Appetitive memory restoration after electroconvulsive shock in the rat. Journal of Comparative and Physiological Psychology, $1974,87,717-723$.

Miller, R. R., \& Springer, A. D. Induced recovery of memory in rats following electroconvulsive shock. Physiology \& Behavior, 1972, 8, 645-651.

Miller, R. R., \& Springer, A. D. Amnesia, consolidation and retrieval. Psychologicol Review, 1973, 80, 69-79.

Miller, R. R., \& Springer, A. D. Implications of recovery from experimental amnesia. Psychological Review, 1974, 81, 470-473.

Spear, N. E. Retrieval of memory in animals. Psychological Review, 1973, 80, 163-194.

SPEAR, N. E. The processing of memories: Forgetting and retention. Hillsdale, N.J: Erlbaum, 1978.

(Received for publication March 13, 1980; revision accepted May 21, 1980.) 